\title{
The blood-brain barrier friend or foe?
}

\author{
Maurizio Scarpa $\cdot$ David Begley
}

Published online: 26 April 2013

(C) SSIEM and Springer Science+Business Media Dordrecht 2013

The concept for this special issue arose during a workshop organised by The Brains for Brain Foundation in March 2012. The Foundation is dedicated to promoting research and improved therapeutics for inherited paediatric neurodegenerative diseases (www.brains4brain.eu). The Foundation was formed 6 years ago in Madrid when approximately 50 basic scientists and clinicians came together to discuss their work. Prior to the meeting the two groups knew little of each other's background. One group were experts in the treatment of lysosomal storage diseases (LSDs) and the other group were experienced basic scientists studying the cerebral microvasculature which forms the blood-brain barrier (BBB). The meeting was convened to enhance an exchange of ideas between these groups. The combined group has now met annually, and participation has grown.

The BBB represents a major barrier to the delivery of therapeutics targeting the CNS, especially protein interventions. Enzyme replacement therapy (ERT) for the LSDs are effective peripherally but ineffective for the CNS, the result of BBB exclusion. Since the brain is just one of the many organs exhibiting storage and pathology in the ERTs, there is a need to include it as a treatment target in combination with peripheral target organs and systems. Confounding CNS pathology is the finding that pathology is also present in the endothelium and cell associations forming the BBB, which ultimately alters BBB function and compounds the pathological burden. Thus, a broad exchange of clinicians, metabolic specialists and basic scientists may enhance treatment concepts in ERTs. The broader impact of this approach for ERTs centers on informing new treatment strategies for more common neurological

\section{Scarpa}

Department of Pediatrics, University of Padova, Via Giustiniani, 3, Padova, Italy

e-mail: maurizio.scarpa@unipd.it

D. Begley $(\square)$

Kings College London, Institute of Pharmaceutical Science,

Franklin-Wilkins Building, 150 Stamford Street,

London SE1 9NH UK

e-mail: david.begley@kcl.ac.uk conditions (e.g., Alzheimer and Parkinson's diseases, Amyotrophic Lateral Sclerosis, etc.), where the challenges of CNS therapy are comparable to those of the LSDs.

This issue of the JIMD presents selected papers derived from the most recent Brains for Brain Symposium, highlighting new approaches for CNS therapy that overcome restrictions and challenges imposed by the BBB. Two distinguished authorities on the BBB, Drs. J. Abbott (doi 10.1007/s10545013-9608-0) and D. Virgintino (doi 10.1007/s10545-0129574-y), provide an insightful overview of the BBB composition, and the role of radial glial cells as the basic vascular network that forms the neuro-vascular architecture. The emerging hypothesis that the choroid plexi may function as structures that protect the BBB against toxic agents is highlighted by a paper describing the role of NF-E2-related factor-2 (NrF2) as a BBB detoxifying factor from the laboratory of Dr. Ek (doi 10.1007/s10545-012-9551-5). The paper by Dr. R. Brady (doi 10.1007/s10545-012-9515-9), the first investigator to describe the potential of $\mathrm{ERT}^{2}$, highlights the utility of small molecules as tools to modify the natural history of CNS progression in LSDs. Keeping with a theme of small molecules, Dr. C. Pontikis and colleagues (doi 10.1007/ s10545-012-9583-x) report that the pharmacological role of cyclodextrin, the only orphan drug available for Niemann Pick $\mathrm{C}$ disease, seems to be related to its binding to the cerebral endothelium and less so to its capacity to cross the BBB, which provides new insight into the mechanism for mobilization and clearance of CNS cholesterol in this disorder.

Moving to a gene therapy/DNA approach, the paper by Dr. G. Baldo and co-workers (doi 10.1007/s10545-012-9530-x) highlights the potential of treating brain storage in mucopolysaccharidosis type I animals via injection of replication defective self inactivating vectors. This approach has shown promise in improving the sensorimotor impairment of this animal model. Additionally, these vectors may provide novel concepts for future gene therapy strategies. Finally, the paper by Dr. J. Papademetriou (doi 10.1007/s10545-012-9534-6) describes an innovative system to deliver high molecular weight molecules to the brain. In this approach, a high molecular weight 
protein (i.e., acid sphingomyelinase, defective in Nieman Pick A and $\mathrm{B}$ disorders) is coupled with an antibody directed against transferrin (TfR) receptors and the intercellular adhesion molecule (ICAM-1) in order to more efficiently target lysosomal enzyme delivery to the brain.

In sum, we feel this group of papers highlights basic concepts and important new advances in CNS drug delivery and approaches toward circumvention of the challenges posed by the BBB interface. As editors of this segment, we hope that the readership of the Journal gains a better understanding of the complexity of the $\mathrm{BBB}$ and the challenges it introduces in achieving efficacious CNS pharmacotherapy for both storage, and many other Mendelian disorders. 